\title{
NOUVELL $\varepsilon$
}

\section{Anticorps neutralisants tri-spécifiques}

\section{Une solution pour l'immunoprophylaxie ou l'immunothérapie anti-VIH?}

Francis Barin, Karl Stefic
Inserm U1259 et université François Rabelais,

Centre national de référence du VIH, laboratoire de virologie, CHU Bretonneau, 2, boulevard Tonnellé, 37044 Tours Cedex, France.

francis.barin@univ-tours.fr
> La diversité et la rapide évolution du virus de l'immunodéficience humaine (VIH) constituent des obstacles majeurs à son contrôle par l'immunité spécifique. La dernière décennie a cependant été marquée par l'obtention d'anticorps monoclonaux humains anti-VIH, qualifiés de largement neutralisants (bNAb, pour broadly neutralizing antibodies) car susceptibles d'inhiber l'infection

$\rightarrow$ Voir la Synthèse de M. Peressin et al. in vitro d'un large $\mathrm{m} / \mathrm{s} \mathrm{n}^{\circ} 1$, janvier 2014, spectre de variants page 69 différents [1-3] $(\rightarrow)$.

Cinq sites de vulnérabilité situés sur les spicules d'enveloppe du VIH-l sont les cibles de ces bNAb (Figure 1). Trois sont situés sur la glycoprotéine de surface gp120: le site de liaison au CD4 (CD4bs, pour (D4-binding site) et deux sites glycopeptidiques localisés dans les régions variables VIV2 ou V3, reposant sur des chaînes de $\mathrm{N}$-glycanes respectivement en positions N160 et N332. Le quatrième correspond à la région proximale de la membrane située dans l'ectodomaine de la glycoprotéine transmembranaire gp4l (MPER, pour membrane-proximal external region). Le dernier site de vulnérabilité regroupe des épitopes également glycopeptidiques impliquant des résidus situés à l'interface entre les gp120 et gp4l. Le caractère protecteur de certains de ces bNAb ayant été prouvé in vivo dans les modèles animaux (souris humanisées et primates), l'idée de les utiliser en prophylaxie, voire en thérapeutique chez l'homme, a fait son chemin et des premiers résultats sont désormais disponibles [3-6]. Ces bNAb ont cependant des limites liées en grande partie à la diversité du virus. En effet, les données de prophylaxie in vivo sont issues de travaux réalisés chez l'animal avec, après administration des anticorps, une épreuve virulente (challenge) effectuée avec une souche virale unique ou, au mieux, un mélange de deux souches sensible(s) in vitro aux anticorps utilisés [7]. Ces souches ne sont bien évidemment pas représentatives de la diversité virale, et aucun bNAb n'étant efficace à $100 \%$ sur l'ensemble des variants, seule une association de bNAb ayant des spectres d'activité complémentaires pourrait assurer une protection optimale $[7,8]$. De manière similaire, les essais d'immunothérapie chez des patients infectés par le VIH à l'aide de ces bNAb montrent clairement qu'ils sont en grande partie efficaces dans la réduction et le contrôle transitoire de la virémie. Toutefois, ils exercent une pression de sélection sur des variants minoritaires pré-existants échappant à la neutralisation qui émergent alors en tant que population virale résistante [3-6]. Ainsi, seule une association de bNAb sélectionnés parmi les plus actifs devrait être utilisée si le développement de cette stratégie thérapeutique se poursuivait.

La génération d'anticorps bispécifiques, constitués de deux fragments Fabl diri-

\footnotetext{
${ }^{1}$ Le fragment Fab est la région de l'immunoglobuline qui contient les domaines variables, VH et VL, reconnaissant l'antigène.
}

gés contre des sites de vulnérabilité différents sur l'enveloppe virale, avait été décrite en 2016 comme une alternative aux associations d'anticorps $[9,10]$. Les travaux rapportés récemment par Xu et al., réalisés au sein des équipes de J Mascola (vaccine research center, $\mathrm{NIH}$, Bethesda, MD) et G Nabel (Sanofi, Cambridge, MA) vont au delà de ce concept, en proposant désormais le développement et l'utilisation d'anticorps neutralisants tri-spécifiques [11]. Les auteurs ont généré des anticorps tri-spécifiques porteurs de paratopes ${ }^{2}$ susceptibles de se lier à trois sites de vulnérabilité différents sur les spicules d'enveloppe du VIH-1. Parmi ceux-ci l'anticorps VRCOl/ PGDM1400-10ع8 a présenté les meilleures performances in vitro en termes d'activité neutralisante et de facilité de production. C'est donc cet anticorps tri-spécifique qui a été testé in vivo afin d'évaluer son activité protectrice.

L'organisation structurale de l'anticorps tri-spécifique est schématisée dans la Figure 2. Il est constitué d'un fragment Fab non modifié provenant de l'anticorps VRCOl qui cible, sur la gpl20, le site de liaison au CD4, et d'un fragment Fab « chimère ». Ce dernier porte deux paratopes: I'un dirigé contre le site de vulnérabilité glycopeptidique de la région V1V2 de la gp120 (PGDM1400), et l'autre contre la région MPER (membrane-proximal

\footnotetext{
${ }^{2}$ Le paratope est la région de l'anticorps qui interagit avec l'épitope et remplit la fonction de reconnaissance de l'antigène.
} 


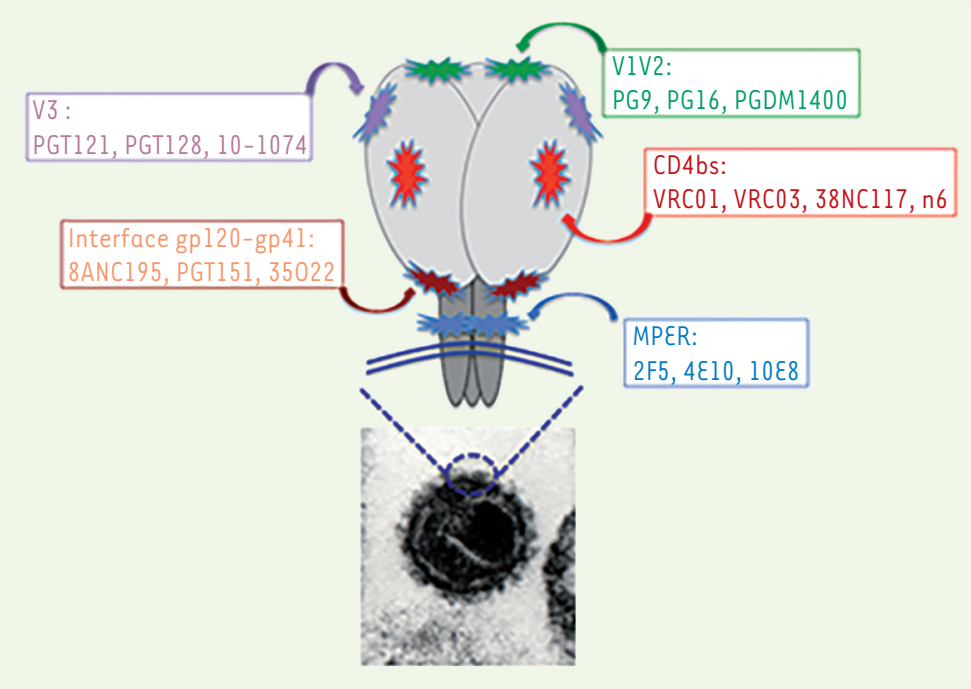

Figure 1. Localisation des cinq sites de vulnérabilité (sites de liaison des anticorps largement neutralisants) sur les spicules d'enveloppe du VIH-1. Cliché de microscopie électronique du VIH-l et «zoom » schématique sur l'organisation des spicules. Ces spicules sont constitués d'un trimère d'hétérodimères (glycoprotéine externe gp120 en gris clair et glycoprotéine transmembranaire gp4l en gris foncé). Les noms des principaux bNAb sont indiqués pour chaque site de vulnérabilité. CD4bs : CD4-binding site; MPER : membrane-proximal external region; VIV2 et V3 : régions variables.

external region) de la gp4l (10ع8). Ce fragment Fab «chimère » est obtenu en positionnant les séquences des régions variables VH des deux anticorps sur le même gène de la chaîne lourde et les séquences correspondantes des régions VL sur le même gène de la chaîne légère. D'après les auteurs, les régions VH de PGDM1400 et 10ع8 doivent être respectivement en position proximale et distale, et l'inverse pour les régions VL. Ces agencements permettent de positionner correctement les domaines pour faciliter l'interaction de chacun des sites $\mathrm{Fv}^{3}$ avec sa cible antigénique (Figure 2). Une modification supplémentaire a été introduite au niveau de la région $\mathrm{Fc}^{4}$ de cet anticorps tri-spécifique. II s'agit de mutations favorisant la liaison au $\mathrm{FcRn}$ (neonatal Fc receptor), augmentant

\footnotetext{
${ }^{3}$ Les domaines variables VH et VL (correspondant au fragment $F v$ ) constituent les régions des anticorps impliquées dans la liaison à l'antigène.

${ }^{4}$ La région $\mathrm{Fc}$ contient les sites de fixation au $\mathrm{Clq}$, le premier composant de la voie classique du complément, et aux différents récepteurs pour la région $F_{c}(R F c)$ ainsi qu'au FcRn.
}

ainsi sa demi-vie lorsqu'il est administré dans l'organisme. Cette stratégie avait déjà été proposée et réalisée pour des bNAb mono-spécifiques [12].

L'activité neutralisante des bNAb est classiquement évaluée selon deux critères: le spectre de neutralisation (breadth) qui correspond au pourcentage de souches virales très diverses neutralisées in vitro, et la puissance (potency) qui correspond à la concentration inhibitrice $\left(\mathrm{Cl}_{50}\right)$. Les résultats obtenus par les auteurs montrent que le spectre de neutralisation de l'anticorps tri-spécifique est supérieur à celui de chacun des trois bNAb. Seulement 4 souches virales parmi un panel de 208 ne sont pas neutralisées par VRCOl/ PGDM1400-10ع8, et la $\mathrm{Cl}_{50}$ moyenne est proche de celle des bNAb monospécifiques les plus puissants. Le spectre de neutralisation correspond donc à ce qui serait attendu pour un effet additif de l'association des trois bNAb parentaux. L'activité protectrice de l'anticorps tri-spécifique a ensuite été testée in vivo en utilisant le modèle animal de référence, des macaques exposés à des virus SHIV (virus chimères SIV [simian immunodeficiency virus] porteurs d'enveloppes du VIH-1). L'anticorps VRC01/PGDM1400-10E8 a été administré par voie intraveineuse à la dose de $5 \mathrm{mg} / \mathrm{kg}$ chez des macaques cinq jours avant l'épreuve virulente, constituée d'un mélange de deux virus SHIV introduit par voie rectale. Ces deux virus avaient été sélectionnés en fonction de leur résistance aux bNAb parentaux, I'un résistant à PGDMl400 (SHIV Bal P4) et l'autre à VRCOl (SHIV 325C). En parallèle deux groupes d'animaux ont reçu en prophylaxie soit PGDMI400 soit VRCO1. Les résultats montrent une protection de tous les animaux ayant reçu l'anticorps tri-spécifique et, au contraire, des échecs de protection pour les animaux ayant reçu uniquement PGDM1400 ou VRCO1. Les données obtenues in vitro et in vivo suggèrent donc que l'anticorps tri-spécifique pourrait être utilisé en prophylaxie de préférence à l'association de trois bNAb monospécifiques.

$\varepsilon n$ plus de l'intérêt premier en terme d'efficacité biologique, les auteurs mettent en avant l'intérêt "logistique » du développement clinique de l'anticorps tri-spécifique, notamment le bénéfice de développer un seul produit biologique plutôt que trois produits différents qui devraient être associés: simplification à la fois de la production industrielle et de l'enregistrement auprès des autorités de santé. Si la preuve de concept est apportée de belle manière, il conviendra désormais d'étudier l'efficacité de l'anticorps dans des essais cliniques chez l'homme ainsi que l'absence d'effets indésirables. L'une des questions majeures à laquelle il faudra répondre concerne l'immunogénicité de ce type de molécule artificielle, l'induction d'une réponse immunitaire antianticorps tri-spécifique risquant d'hypothéquer son utilisation au long cours. Il conviendra également de surveiller l'évolution de la sensibilité du VIH-l à la 


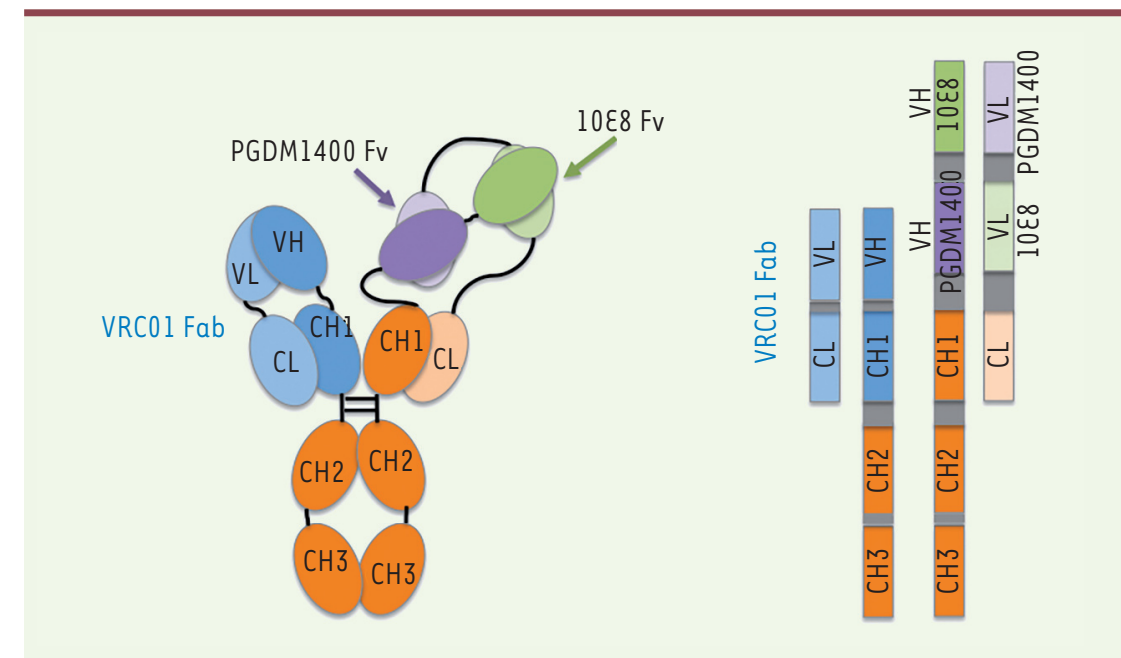

Figure 2. Organisation structurale de l'anticorps tri-spécifique VRC01/PGDM1400-10ع8 (illustration adaptée de Xu et al. [11]).

neutralisation par ces anticorps, le virus, en tant qu'espèce, semblant dériver vers une plus grande résistance à la neutralisation depuis son introduction dans la population humaine [8]. Le développement de molécules anti-virales à action ou libération prolongée (cabotégravir, rilpivirine, etc.) pourrait également constituer une limite au développement des bNAb ou d'anticorps tri-spécifiques en prophylaxie du fait d'un très probable coût de production inférieur à celui des immunoglobulines et de conditions de conservation et d'approvisionnement moins contraignantes.

Quoi qu'il en soit, ce travail vient à nouveau illustrer l'inventivité développée pour lutter et tenter de contrôler I'infection par le VIH. Les anticorps neutralisants occupent désormais une place

\section{RÉFÉRENCES}

1. Mouquet H. Antibody B cell responses in HIV-1 infection. Trends Immunol 2014 ; 35 : 549-61.

2. Burton D, Mascola JR. Antibody responses to envelope glycoproteins in HIV-1 infection. Nat Immunol 2015; $16: 571-6$.

3. Caskey M, Klein F, Lorenzi JCC, et al. Viraemia suppressed in HIV-l-infected humans by broadly neutralizing antibody 3BNC117. Nature $2015 ; 522$ : 487-93.

4. Peressin M, Holl V, Moog C. Les anticorps anti-VIH : de multiples activités antivirales. Med Sci(Paris) 2014 ; $30: 69-75$.

5. Lynch RM, Boritz $\varepsilon$, Coates $\varepsilon \varepsilon$, et al. Virologic effects of broadly neutralizing antibody VRCOl administration during chronic HIV-1 infection. Sci Transl Med 2015 ; $7: 319$ ra206.

6. Scheid JF, Horwitz JA, Bar-On Y, et al. HIV-1 antibody $3 \mathrm{BNCl17}$ suppresses viral rebound in humans during treatment interruption. Nature 2016 ; 535 : 556-60.

7. Julg B, Liu PT, Wagh K, et al. Protection against a mixed SHIV challenge by a broadly neutralizing antibody cocktail. Sci Transl Med 2017 ; 9 : eaao4235

8. Bouvin-Pley M, Morgand M, Moreau A, et al. Evidence for a continuous drift of the HIV-l species towards higher resistance to neutralizing antibodies over the course of the epidemic. PLoS Pathog 2013 ; 9 : el003477.

9. Bournazos S, Gazumyan A, Seaman MS, et al. Bispecific anti-HIV-l antibodies with enhanced breadth and potency. Cell $2016 ; 165$ : 1609-20.

10. Huang $\mathrm{Y}, \mathrm{Y}_{\mathrm{u}} \mathrm{J}$, Lanzi $\mathrm{A}$, et al. Engineered bispecific antibodies with exquisite HIV-1-neutralizing activity. Cell $2016 ; 165$ : 1621-31.

11. Xu L, Pegu A, Rao $\varepsilon$, et al. Trispecific broadly neutralizing HIV antibodies mediate potent SHIV protection in macaques. Science $2017 ; 358: 85-90$.

12. Ko SY, Pegu A, Rudicell RS, et al. Enhanced neonatal Fc receptor function improves protection against primate SHIV infection. Nature $2014 ; 514$ : 642-47.

13. Ekiert DC, Friesen RHE, Bhabha G, et al. A highly conserved neutralizing epitope on group 2 influenza $A$ viruses. Science 2011 ; 333 : 843-50.

14. Rouvinski A, Guardado-Calvo P, Barba-Spaeth $G$, et al. Recognition determinants of broadly neutralizing antibodies against dengue viruses. Nature $2015 ; 520$ : 109-13.

15. Kadam RU, Juraszek J, Brandenburg B, et al. Potent peptidic fusion inhibitors of influenza virus. Science 2017 ; $358: 496-502$.

\section{LA FONDATION PREMUP : UN OPÉRATEUR DE TERRAIN EN PÉRINATALITÉ RECONNU POUR SON EXCELLENCE \&T SON INTERDISCIPLINARITÉ}

La Fondation de coopération scientifique PremUp, unique en Europe, intervient sur la prévention du handicap à la naissance, par la protection de la santé de la femme enceinte et du nouveau-né.
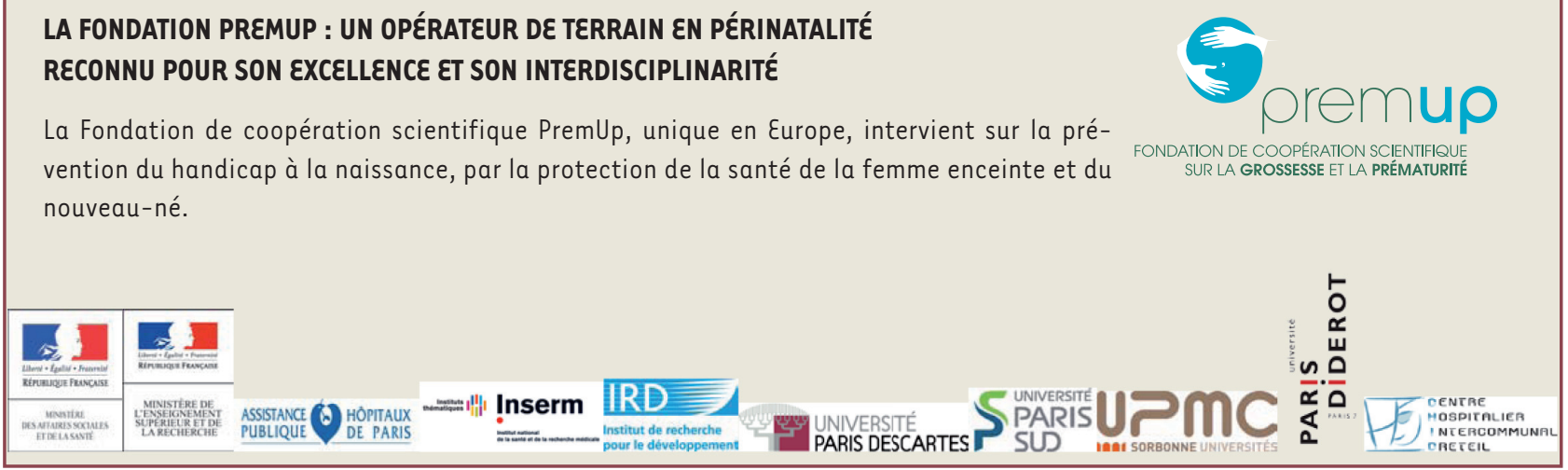